\title{
A catalogue of average magnetic phase curves
}

\author{
V.D. Bychkov ${ }^{1}$, L.V. Bychkova ${ }^{1}$ and J. Madej ${ }^{2} \dagger$ \\ ${ }^{1}$ Special Astrophysical Observatory, Russian Academy of Sciences, Nizhnij Arkhyz, Russia \\ email: vbych@sao.ru \\ ${ }^{2}$ Warsaw University Observatory, Al. Ujazdowskie 4, 00-478 Warszawa, Poland \\ email: jm@alkor.astrouw.edu.pl
}

\begin{abstract}
We collected published measurements of the effective magnetic fields of stars on the Main Sequence and above it, and compliled a catalogue of periodic $B_{\mathrm{e}}$ variations. We present magnetic phase curves for 139 stars and tables of their parameters. Most of catalogued objects are chemically peculiar A and B type stars (134 stars).
\end{abstract}

Keywords. Catalogs, stars: magnetic fields, stars: variables: other

\section{Introduction}

Variability of the effective magnetic fields $B_{\mathrm{e}}$ in Ap stars was discovered over 50 years ago (Babcock \& Burd 1952). A large amount of observational data has been collected since then. We performed an extensive literature search and selected all available measurements of the effective (longitudinal) magnetic field $B_{\mathrm{e}}$ for Main Sequence and a few other stars. Average magnetic phase curves $B_{\mathrm{e}}(\phi)$ were constructed for those stars for which we know magnetic (i.e., rotational) periods or could determine them.

A star with a large-scale magnetic field can be described by the oblique rotator model, in which the axis of a magnetic dipole is inclined to the rotation axis. The dipole field itself does not change with time. Periodic variability of the effective magnetic field $B_{\mathrm{e}}$ is caused by changes of aspect due to stellar rotation. Therefore the period of magnetic $B_{\mathrm{e}}$ variations, $P_{\text {mag }}$, can be identified with the rotational period $P_{\text {rot }}$.

This model was proposed to explain the behavior of magnetic CP stars, which exhibit periodic variations of $B_{\mathrm{e}}$ (Stibbs 1950, Preston 1967). In that model, the dipole magnetic field is frozen into the stellar atmosphere, and is intrisically constant at each point.

Our catalog presents average magnetic phase curves in a homogeneous form. We have also determined other parameters of magnetic variability for all stars which are briefly described below.

\section{Parameters of magnetic variability}

In this section we list the parameters of the magnetic phase curves, and also the parameter $r$, which was defined by Stibbs (1950). These are: $B_{0}, B_{1}, B_{2}, T_{0}, P$ and $r$.

\subsection{Sine wave}

For all stars with an adequate number of $B_{\mathrm{e}}$ determinations and for which the period of magnetic variability $P_{\text {mag }}$ was known, we have determined the best fit for the relation of $B_{\text {e vs. phase } \phi}$

$$
B_{e i}(\phi)=B_{0}+B_{1} \cos \phi,
$$

$\dagger$ Present address: SAO RAS, Nizhnij Arkhyz, 369167 Russia. 


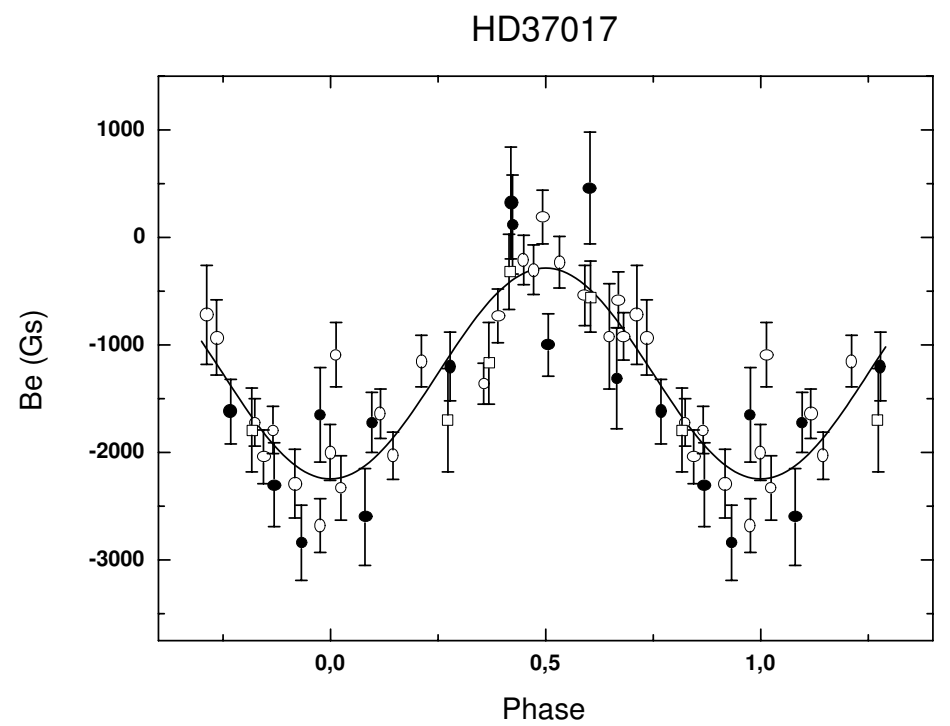

Figure 1. Sample phase curve: single wave for HD 37017. Open circles: Bohlender et al. (1987) (H lines), filled circles: Borra \& Landstreet (1979) (H lines), open squares: Bohlender et al. (1987) (He $\lambda 5867)$.

where

$$
\phi=2 \pi\left(\frac{T_{i}-T_{0}}{P}\right) .
$$

by the least squares method. Here $B_{0}$ is the average field, $B_{1}$ stands for half of the amplitude, $T_{i}$, the time of measurement, $P$, the the period, and $T_{0}$ is the zero epoch, i.e., time corresponding to the zero phase $\phi$. We have chosen the zero epoch $T_{0}$ so that the phase $\phi=0$ corresponds to the minimum of the best fit magnetic curve for all stars.

\subsection{Parameter $r$}

Parameter $r$ relates both angle $\beta$ between the magnetic dipole axis and the rotational axis, and the angle $i$ between the rotational axis and the line of sight

$$
r=\frac{\cos \beta \cos i-\sin \beta \sin i}{\cos \beta \cos i+\sin \beta \sin i} .
$$

Alternatively, one can write

$$
r=\frac{B_{\mathrm{e}}(\min )}{B_{\mathrm{e}}(\max )} .
$$

\subsection{Double wave}

If the shape of the magnetic phase curve is more complex than a simple cosine, we include the second harmonic

$$
B_{e i}(\phi)=B_{0}+B_{1} \cos \left(\phi+z_{1}\right)+B_{2} \cos \left(2 \phi+z_{2}\right) .
$$




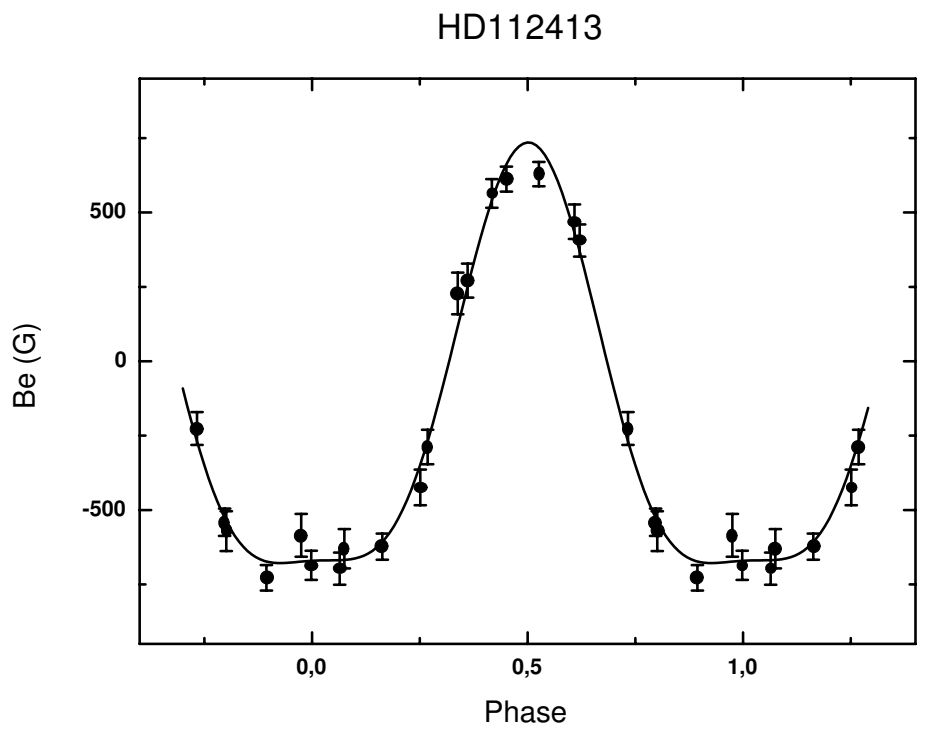

Figure 2. Sample phase curves: double wave. The HD 112413 phase curve $B_{\mathrm{e}}(\phi)$ derived from $B_{\mathrm{e}}$ measurements of Wade et al. (2000) (metal lines, LSD method). The average phase curve $B_{\mathrm{e}}(\phi)$ has the form of a double wave.

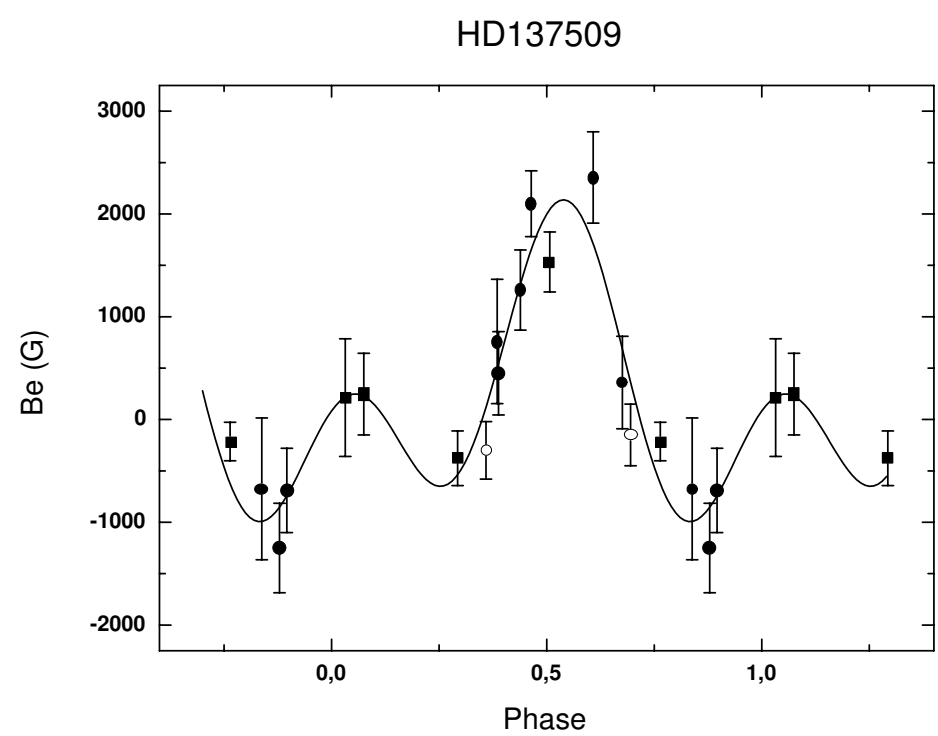

Figure 3. Sample phase curves: double wave for HD 137509. The magnetic phase curve is described best by a double wave. Filled squares, Mathys G.\& Hubrig (1997); open circles, Bohlender et al. (1993); filled circles, Mathys (1991). 


\section{Error analysis}

For each star in the sample we performed a $\chi^{2}$ test to evaluate the goodness of the assumed fit, given either by Eq. 1 or Eq. 4, and estimated the scatter of the available $B_{\mathrm{e}}$ measurements. The $\chi^{2}$ statistical test can indicate a large discrepancy between observed points and the assumed fitting curve if either the fitting curve is intrinsically inconsistent with observations, or the errors of observations (i.e. values of $B_{\mathrm{e}}$ ) are overestimated.

Error estimates of all parameters $T_{0}, B_{0}, B_{1}, B_{2}$, and $r$, were performed as follows. For each $B_{\mathrm{e} i}$ measurement with known standard error $\sigma_{i}$ we generated a series of secondary $B_{\mathrm{e} i}^{\mathrm{sec}}$ values with a random number generator. The values of $B_{\mathrm{e} i}^{\mathrm{sec}}$ had a normal distribution around the observed $B_{\mathrm{e}} i$ with the width $\sigma_{i}$. (When the authors did not provide $\sigma_{i}$ estimate, we used an arbitrarily error typical for a given method of observation.) This method generated set of artificial values of $B_{\mathrm{e}}$, for which the secondary parameters $T_{0}$, $B_{0}, B_{1}, B_{2}$, and $r$ were determined.

These computations were repeated usually 1000 times or more. In this way we obtained numerous sets of fitting parameters, and were able to estimate errors of $T_{0}, B_{0}, B_{1}, B_{2}$, and $r$ separately.

\section{Tabular data}

A complete catalog of the investigated stars and the corresponding parameters of the best fit sine magnetic curves (Eq. 2.1) is presented in Table 1, which will be available on the Internet. Table 1 lists the HD number, $B_{0}, \sigma_{B_{0}}, B_{1}, \sigma_{B_{1}}$, the period $P, T_{0}, \sigma_{T_{0}}, N$ (the number of individual points, $\sigma$ (the average scatter of $B_{\mathrm{e} i}$ about the fitting curve), $r, \sigma_{r}$, the value of $\chi^{2}$ (for one degree of freedom), reference number, and (in some cases) the running number of brief comments.

Table 2 of our catalog presents for each star: the HD number, parameter $r$ and its standard error $\sigma_{r}, N_{V}=N-3$ or $N-5$ (for sine wave. or double wave fits, respectively), $\chi^{2}$ for one degree of freedom, method and reference numbers.

There are 18 magnetic stars which display more complex phase curves $B_{\mathrm{e}}(\phi)$. For them phase curves were fitted by an expansion in the harmonic series with the second cosine term, see Eq. 2.5 (double wave).

Table 3 presents additional parameters necessary to define the double wave. These are coefficients $B_{2}$, phase shifts $z_{1}$ and $z_{2}$, and their errors $\sigma_{B_{2}}, \sigma_{z_{1}}, \sigma_{z_{2}}, N_{V}$, values of $\chi^{2}$, number of references and comments. Here $N_{V}=N-5$.

\section{Summary}

We compiled a catalog of magnetic phase curves $B_{\mathrm{e}}(\phi)$ for 139 stars, which exhibit periodic variations of the effective magnetic field $B_{\mathrm{e}}$. Most catalogued objects, 134 stars, are chemically peculiar A and B type stars. The catalog consists of figures, which display individual $B_{\mathrm{e}}$ measurements and error bars, and phase curves approximated either by a sine wave, or by a double wave.

The catalog presents lists the following parameters of the magnetic phase curves: coefficients $B_{0}, B_{1}$, and $B_{2}$ of the harmonic expansion of $B_{\mathrm{e}}(\phi)$, the period $P$ (in days) and the Julian Day of zero phase $T_{0}$, and the coefficient $r$ defined by Stibbs (1950). 


\section{Acknowledgements}

Our research is based on data compiled and posted in the SIMBAD, ADS, and CDS databases. We acknowledge support from the Polish Committee for Scientific Research grant No. 1 P03D 00126.

\section{References}

Babcock, H.W., Burd, S. 1952, ApJ 116, 8

Bohlender D.A.,Brown D.N.,Landstreet J.D., Thompson I.B. 1987, ApJ 323, 325

Bohlender D.A.,Landstrret J.D.,Thompson I.B. 1993, A\& A 269, 355

Borra E.F. \& Landstreet J.D. 1979, ApJ 228, 809

Bychkov, V.D., Bychkova, L.V., Madej, J. 2004,A\&A in press

Mathys G. 1991, A\&SASS 89, 121

Mathys G. \& Hubrig S. 1997, A\&SS 124, 475

Preston G.W. 1967, ApJ 150, 547

Stibbs, D.W. 1950, MNRAS 110, 395

Wade G.A.,Donati J.-F.,Landstreet J.D.and Shorlin S.L.S. 2000, MNRAS 313, 851 